\title{
QCD Form Factors and Hadron Helicity Non-Conservation
}

\author{
John P. Ralston ${ }^{1}$ and Pankaj Jain ${ }^{2}$ \\ ${ }^{1}$ Department of Physics \& Astronomy \\ University of Kansas \\ Lawrence, KS 66045 \\ ${ }^{2}$ Physics Department \\ I.I.T. Kanpur, India 208016
}

\begin{abstract}
Recent data for the ratio $R(Q)=Q F_{2}\left(Q^{2}\right) / F_{1}\left(Q^{2}\right)$ shocked the community by disobeying expectations held for 50 years. We examine the status of perturbative QCD predictions for helicity-flip form factors. Contrary to common belief, we find there is no rule of hadron helicity conservation for form factors. Instead the analysis yields an inequality that the leading power of helicity-flip processes may equal or exceed the power of helicity conserving processes. Numerical calculations support the rule, and extend the result to the regime of laboratory momentum transfer $Q^{2}$. Quark orbital angular momentum, an important feature of the helicity flip processes, may play a role in all form factors at large $Q^{2}$, depending on the quark wave functions.
\end{abstract}




\section{The Nature of High Energy Reactions}

There is an asymmetry in high energy reactions due to the Lorentz transformation. The spatial coordinate parallel to the boost axis is Lorentz contracted. The momentum fraction $x$ of partons inside hadrons is thereby distributed over the entire possible range $0<x<1$. This phenomenon is dynamical, because a boost of interacting fields is dynamical, and the $x$ dependence of wave functions cannot reliably be calculated in perturbative QCD. Instead, the $x$-dependence of wave functions is extracted from experimental data.

Meanwhile the transverse spatial coordinate $\vec{b}$ is Lorentz invariant. The transverse coordinate has a certain calculability via perturbative QCD. There is a great deal of interest and controversy associated with the transverse coordinate in exclusive reactions. The transverse spatial coordinate can be probed in reactions sensitive to the angular momentum flow. In some reactions, the sum of the helicities going into a hadronic reaction is automatically conserved. This is the case of the proton's Dirac form factor $F_{1}$ in the high energy limit. When the sum of the helicities is not conserved, angular momentum conservation requires either extra constituents, or quark orbital angular momentum. This is the case of $F_{2}$, the proton's Pauli form factor.

The Jefferson Laboratory has observed $Q F_{2}\left(Q^{2}\right) / F_{1}\left(Q^{2}\right) \sim$ const. up to the highest values of $Q^{2} \sim 5.8 \mathrm{GeV}^{2}$ yet measured [1, 2]. The data was initially very surprising, and the field may have reached a pivotal point in comparison with the quark model. For a long time it was held sacred that $Q^{2} F_{2} / F_{1} \sim$ const at large $Q^{2}$. This rule appears to predate QCD. It has ancient origins in renormalization questions involving protons as elementary fundamental fields 3 .

Meanwhile a perturbative QCD model assuming non-zero quark orbital angular momentum (OAM) predicted $Q F_{2}\left(Q^{2}\right) / F_{1}\left(Q^{2}\right) \sim$ const 4, 5. A relativistic quark model prediction [6] fits the flatness of $Q F_{2}\left(Q^{2}\right) / F_{1}\left(Q^{2}\right)$ equally well. These papers countered the ancient wisdom, because they shared the common feature of quark orbital angular momentum in the wave functions.

The hypothesis of zero OAM sometimes appeals to the non-relativistic quark model in the rest frame. Yet very little of high energy physics and pQCD starts in the rest frame. The perturbative quark wave functions are unrestricted in angular momentum content, except for Lorentz symmetry. Observation of non-zero quark OAM is a leading candidate to resolve the 
proton "spin crisis", which is the fact that the sum of the perturbative quark spins does not equal the spin of the proton. Consequently the Jefferson lab's data has even broader implications than the mystery of large $Q^{2}$ form factors.

Here we address whether $Q F_{2}\left(Q^{2}\right) / F_{1}\left(Q^{2}\right) \sim$ const. is a transient feature of comparatively low energy experiments, or a fact destined to persist at higher $Q^{2}$. If the flatness of this ratio is due to quark OAM, will the ratio stay flat with increasing $Q^{2}$ ? The question leads us to re-examine the roots of the "hadron helicity conservation" rule [7. We find that pQCD itself is rather neutral, and only gives an inequality between the powers governing helicityflip processes and helicity conserving ones. As a result $Q F_{2} / F_{1} \sim$ const. may extend to arbitrarily large values of $Q^{2}$, without violating anything sacred.

\section{Definitions and Discussion}

Orbital angular momentum: By quark orbital angular momentum (OAM) we refer to an expansion in $S O(2)$ representations (commonly known as $L_{z}$ states) with quantization axis aligned along the particle 3 -momentum $\vec{P}$. The eigenstates of $L_{z}$ are invariant under boosts along the $z$-axis. We do not use spherical harmonics, and we treat the longitudinal coordinates as scaling variables. Let the transverse spatial coordinate be $\vec{b}=b(\cos \phi, \sin \phi)$. We expand operators or wave functions as

$$
\psi=\sum_{m} e^{i m \phi} \psi_{m}(x, b)
$$

where $x$ is the Feynman light-cone fraction of $z$ (or "+") momentum. ${ }^{1}$ OAM can also be re-expressed with generalized parton distributions in a manifestly gauge-invariant formalism [8, 9].

Chirality versus Helicity: The proton's Pauli form factor $F_{2}$ contains information on the orbital angular momentum of the quarks, but it is indirect. Strictly speaking, the amplitude $i \bar{u}\left(p^{\prime}, s^{\prime}\right) F_{2}\left(Q^{2}\right) \sigma^{\mu \nu} q_{\nu} u(p, s)$ represents the amplitude for chirality of the proton to flip under momentum transfer $Q$. The chirality (eigenvalue of $\gamma_{5}$ ) of light quarks flips very little in pQCD

\footnotetext{
${ }^{1}$ Of course perturbative wave functions are theory constructs, and fields at finite separation are technically not gauge invariant, in general. Observables are nevertheless gauge invariant when all the legs of diagrams are contracted properly.
} 
reactions, by about $m_{q} / Q<<1$, for quarks of mass $m_{q} \sim$ few $M e V$. The chirality of Fermions is proportional to their helicity, with corrections of order $m / Q$. Adding another constituent to the scattering is also down by $O\left(1 / Q^{2}\right)$. Then by this reasoning, at large $Q^{2}>G e V^{2}$, it is not possible to flip either the chirality or helicity of the proton with a virtual photon, unless there is internal quark orbital angular momentum present to satisfy the selection rules.

What is Quark Counting? We separate the quark-counting model of Brodsky, Farrar, and independently Matveev et al [10] from the asymptotic short distance (asd) model of Brodsky and Lepage [11, 12. The earlier theory is one of counting propagators and the number of scattered constituents. The latter theory is much more detailed, imposing a certain factorization of the hard reaction into components made from the $s$-wave, $m=0$ BetheSalpeter wave functions. This does not come by listing all diagrams, and is not a feature of the starting theory. Instead the framework is developed by assuming the framework and classifying terms within the assumptions. The asd approach is characterized by taking the zero-distance limit in the first step, and replacing the rest of the calculation by integrals over Feynman $x$ fractions using "distribution amplitudes", or similar quantities with no transverse information. However the Feynman rules instruct us to leave the longitudinal and transverse integrals coupled. Perform the integrals, and afterwards take the limit of large $Q$ (if wanted). If the two limits are not the same (and they are not in general), then the asd assumptions can fail.

The transverse coordinates are gone in the asd approach, being evaluated within $1 / Q$ of zero. HHC follows instantly as a test of the framework, independent of the wave functions used. By omitting OAM $m \neq 0$, the prediction for $F_{2}=0$, and one cannot recover any prediction for finite $F_{2}$ in the formalism. As far as we know, all asd predictions of $F_{2}$ are indirect and deduced by elimination: since $F_{1} \sim 1 / Q^{4}$, and $Q F_{2}$ could not be calculated, then $F_{2} \sim F_{1} / Q^{2}$ must lie in the detritus not calculated. A recent calculation [13], however, finds that within the framework of a generalized asd model $F_{2} \sim 1 / Q^{6}$. It is also possible to modify the model by including quark mass effects [14. Our goal here is to understand the limit of arbitrarily light quarks and neglecting effects of order $m_{q} / Q$.

We separated quark counting from asd because the two are not the same theory. Should one believe either? There are good indications from the 
scaling laws that quark-counting has some truth. We have no religious commitment here, and the possibility that a fraction of the amplitude is due to "soft physics" must be given credit [15, 16. But amplitudes cannot all be soft, because the form factors are not seen to fall exponentially with $Q$. Indeed the quark-counting scaling laws generally work well. Meanwhile the inapplicability of $\mathrm{HHC}$ to $F_{2}$ or any other helicity flip reactions [17] shows we cannot use asd.

Regarding Generalized Parton Distributions: Generalized parton distributions $(G P D)$ appear to be an ideal way to proceed. Lorentz covariance can be used to set up matrix elements and expressions for the form factor in an apparently model-independent way. As far as we know, form factors were the first instance of GPD, used in the paper of Soper [18 in 1977, which also contains a transformation to a particular transverse spatial coordinate. We will have occasion to revisit the conclusions of that paper in Section 3.7.

Despite our GPD-based predictions [8, 4, 5] for ratios and the welcome rediscovery of $G P D$ in the field, we chose not to make them the vehicle for this analysis. The reason is that GPD are so general they do not immediately contain the information there are three quarks in the proton. To incorporate the information one can start with wave functions and integrate out all but two quark legs. Since our concern is precisely these integrations, we would have nothing to gain. We caution the reader, in any event, that the method of approximating integrals by asd methods will have similar limit-interchange problems when clothed in GPD language. Diehl et al. [19] discuss general relations, and their formulas codify a relation of $F_{2}$ to quark OAM.

Some Familiar, But Inexact Assertions: Suppose we have non-zero OAM in the wave functions and we try to use the assertion of the asd approach,

$$
\Delta b \Delta Q_{T}>1
$$

Here $\Delta b$ is the resolved transverse quark separation, in a frame where the momentum transfer $Q \sim \Delta Q_{T}$ is transverse. We have written the relation like the uncertainty principle, to give it a chance to be seductive. We observe next that, merely from continuity, a wave function $\psi_{m}$ carrying $m$ units of angular momentum scales like $b^{m}$ as $b \rightarrow 0$. Then under these assumptions each unit 
of orbital angular momentum of the quarks will lead to amplitudes suppressed by a corresponding power of $1 / Q$ at large $Q$. This familar assertion has been repeated endlessly in the literature, yet we will show that this type of counting does not represent QCD. It is seductive but it is not right.

Our Approach: To address $F_{2}$ properly, one must go beyond short-distance to restore the transverse coordinate. This is called "impact-parameter factorization" [20, 21]. This well-justified method has dominated recent attention in perturbative QCD [22, 23, 24, 25, 26]. In impact-parameter factorization the form factor is written as

$F\left(Q^{2}\right)=\int\left(\Pi d x_{i} d^{2} k_{T, i}\right)\left(\Pi d x_{i}^{\prime} d^{2} k_{T, i}^{\prime}\right) \bar{\psi}\left(x, k_{T}\right) H\left(x_{i}, x_{i}^{\prime}, k_{T, i}, k_{T, i}^{\prime} ; Q\right) \psi\left(x^{\prime}, k_{T}^{\prime}\right)$.

The impulse approximation has been used to set the light-cone "time" to zero. There are no a-priori assumptions in Eq. 2 about short-distance. If Sudakov effects are used consistently, then wave functions in Eq. 2 concentrate the dominant region into one which is perturbatively calculable, without assuming zero-distance as a starting point. We do not go to the further extreme of Ref. [22] towards asserting that the ultimate output of Sudakov effects is the asd model. We find this unjustified. To get that result one needs assumptions about the wave functions that are simply not known.

\section{Calculations}

Here we present pQCD calculations and also illustrate certain features that are even more general.

\subsection{Role of The Transverse Coordinate}

Return to the general expression Eq. 2] To leading order and neglecting transverse momentum in Fermion numerators, the hard scattering depends on differences $\vec{k}_{T}-\vec{k}_{T}^{\prime}$ before and after the hard collision. The importance of this variable seems rather general, because the sum of the transverse momenta are conjugate to the overall spatial location of the hard scattering, which by translational invariance drops out. ${ }^{2}$ In a process with a single hard

\footnotetext{
${ }^{2}$ From translational invariance, the hard kernel must depend on the difference of space coordinates. The transverse separation $b$ is only one such difference, and the one of interest here. Other differences such as the longitudinal ones also occur.
} 
exchange (a pion or meson form factor) the transverse integrals take the form of convolution:

$$
\begin{array}{r}
F(Q)=\int d^{2} k_{T} f\left(k_{T}\right) \bar{\psi}\left(\vec{k}_{T}\right) ; \\
f\left(k_{T}\right)=\int d^{2} k_{T}^{\prime} H\left(k_{T}-\vec{k}_{T}^{\prime} ; Q\right) \psi\left(\vec{k}_{T}^{\prime}\right), \\
=\int \frac{d^{2} b}{(2 \pi)^{2}} e^{i \vec{k}_{T} \cdot \vec{b}} \tilde{H}(b ; Q) \tilde{\psi}(b, x) .
\end{array}
$$

The $x$ variables were suppressed. Consequently the expressions are diagonal in $b$, with

$$
\begin{array}{r}
F(Q)=\int \frac{d^{2} b}{(2 \pi)^{2}} d x d x^{\prime} \tilde{\psi}^{*}(b, x) \tilde{H}\left(b ; x, x^{\prime}, Q\right) \tilde{\psi}\left(b, x^{\prime}\right), \\
=\sum_{m m^{\prime} m^{\prime \prime}} \int \frac{d^{2} b}{(2 \pi)^{2}} d x d x^{\prime} d \phi e^{-i\left(m-m^{\prime}-m^{\prime \prime}\right) \phi} \tilde{\psi}_{m}^{*}(b, x) \tilde{H}_{m^{\prime}}\left(b ; x, x^{\prime}, Q\right) \tilde{\psi}_{m^{\prime \prime}}\left(b, x^{\prime}\right) .
\end{array}
$$

All contributions to OAM are explicit at this stage. Expansion of the hard scattering $H_{m}$ was introduced because the kernel can also carry angular momentum and be anisotropic, via the direction of the hard momentum $\vec{Q}$. The selection rules conserving angular momentum will come from the $\phi$ integrals.

\subsection{Gluon Exchange Kernel}

Consider the simplest one-gluon approximation to the kernel, $H=4 \pi C_{F} \alpha_{s} / q^{2} ; q^{\mu}=$ $x P^{\mu}-x^{\prime} P^{\mu^{\prime}}+\vec{k}_{T}^{\mu}-\vec{k}_{T}^{\mu^{\prime}}$ ( Fig 1). This is written out as

$$
\begin{aligned}
H\left(\vec{k}-\vec{k}_{T}^{\prime} ; x, x^{\prime}, Q\right) & =4 \pi C_{F} \alpha_{s} \frac{1}{x x^{\prime} Q^{2}+\left(\vec{k}_{T}-\vec{k}_{T}^{\prime}\right)^{2}} \\
\tilde{H}\left(b ; x, x^{\prime}, Q\right) & =8 \pi^{2} C_{F} \alpha_{s} K_{0}\left(\sqrt{x x^{\prime} Q^{2} b^{2}}\right)
\end{aligned}
$$

When integrated with non-singular functions of $b$ and $x$, the dominant region is not determined by Eq. 1, but instead the Bessel function restricts to

$$
\sqrt{x x^{\prime}} Q b<1
$$

Clearly Eq. 6 is more accurate than Eq. 1, because the partons entering the reaction carry $x P$, rather than $P$ and can only be scattered through $x x^{\prime} Q^{2}$ 
momentum transfer-squared. This has been recognized for a long time, and common wisdom ascribes an average value of $x \sim 1 / 3$ for valence quarks. It is commonly accepted that consistent appearance of $x x^{\prime} Q^{2}$ postpones any onset of short-distance dominance to higher values of $Q^{2}$ compared to the naive implications of Eq. 1 .

A Physics Question: We pause to question how the "uncertainty principle" of Eq11, $b \sim 1 / Q$, could have misled the field. First, it was not the uncertainty principle, but a guess at dominant regions. Somehow $x$, from the longitudinal coordinate, got into a relation between transverse things. We seek a physical explanation.

Suppose we stand near a fast moving charge with energy $E$, mass $m$, at impact parameter $b$. Relativity predicts a pulse of fields with a time scale $\Delta t \sim b / \gamma^{3}$ Since $\gamma=E / m$ the fields depend on $\Delta t \gamma / b$. The partons are spread over $p_{z}=x E \sim 1 / \Delta t$. Therefore the fields depend on $(m x b)$. This explains how the longitudinal fraction inserted itself. In a perturbative transition amplitude between $x, x^{\prime}$ values, dependence on $\sqrt{x x^{\prime}} Q b$ is generic, and nearly kinematic. The time-scale dependence on impact parameter has been identified as important. The time-scale $(x)$ dependence is of course set by the non-perturbative part of the problem, the $x$ dependence of wave functions.

\subsection{Time Scale Smearing: Interplay of $x$ and $b$}

Exploring the integrals, the $b^{|m|} \sim 1 / Q^{|m|}$ counting will still occur, from dimensional analysis, after doing the $b$ integrations in each $x, x^{\prime}$ integration bin. It looks like Eq. 1 and its conclusions will win after all. What is quite surprising, and highlighted here, is that there is no simple rule after the $x, x^{\prime}$ integrations are done. The scaling indicated by dimensional analysis is "erased" by the $x$-integrals under broad conditions. The physical origin, of course, is that the dynamical time scale distribution in the wave functions ( $x$ distributions) is not something we are priviledged to predict. The $x$-distribution is set by the proton itself in the quiet of vacuum over infinite time. The "time-scale smearing" destroys naive use of the uncertainty prin-

\footnotetext{
${ }^{3}$ This time scale is also a Lorentz-contracted pancake longitudinal distance scale. Our use of "time-scale" is consistent with the impulse approximation.
} 
ciple, and its counting of powers. However it does not destroy the use of pQCD, which always contains integration over $x$.

\subsection{The Dominant Power of $x$}

It suffices to use the kernel Eq. 5 to show the effects. Since our focus is the orbital angular momentum, we consider the integrals with factors of $b^{|m|}$ explicit. The selection rules from the angular integrals $e^{-i\left(m-m^{\prime}-m^{\prime \prime}\right) \phi}$

are obvious. We use a Gaussian function $\tilde{\Phi}(b, x, \zeta)=b^{A} e^{-b^{2} /\left(2 a^{2}\right)} \tilde{\phi}(x, \zeta)$ to represent the wave functions cutting off large $b$, as well as a Sudakov model, described below. The factor of $b^{A}$ is the phase-space to find $A$ quarks close together from naive quark-counting. We change variables for the longitudinal fractions to

$$
\begin{gathered}
x=\sqrt{x x^{\prime}}, \\
\zeta=x / x^{\prime} .
\end{gathered}
$$

We parameterize

$$
\tilde{\phi}_{m}(x, \zeta) \sim x^{r+1}(1-x)^{r+1} \phi(\zeta)
$$

as $x \rightarrow 1$ : the $\zeta$ dependence can be left unspecified. For later use parameter $r$ is called the "dominant power of $x$ ". In discussing the region $x \rightarrow 1$, we can expand $\tilde{\phi}_{m}(x, \zeta)$ in a power series. Knowing the dominant power is all that is needed for the arguments to go through.

\subsection{Mellin Method}

We study the large- $Q$ asymptotics by calculating a Mellin transform $F(N)$ conjugate to $Q^{N}$ :

$$
\begin{aligned}
F(N) & =\int_{0}^{\infty} \frac{d Q}{Q} Q^{N} F(Q) \\
& =\int_{0}^{\infty} d b b^{|m|+A+1} \int_{0}^{1} d x \int d \zeta \int_{0}^{\infty} \frac{d Q}{Q} Q^{N} K_{0}(x b Q) \tilde{\Phi}(b, x, \zeta) .
\end{aligned}
$$

The $Q$ integral is carried out easily,

$$
\int \frac{d Q}{Q} Q^{N} K_{0}(x b Q)=2^{-2+N}(x b)^{-N}\left[\Gamma\left(\frac{N}{2}\right)\right]^{2} .
$$


Note that $b^{-N}$ emerges just as expected from dimensional analysis. If we stopped here, then $b^{m}$ would be suppressed by $Q^{-m}$.

The other integrals are then done as

$$
\begin{aligned}
& \int_{0}^{\infty} d b \int_{0}^{1} d x(b x)^{-N} b^{|m|+A+1} x^{r+1}(1-x)^{r+1} e^{-b^{2} /\left(2 a^{2}\right)}= \\
& \frac{2^{\frac{N+m+A-4}{2}} a^{m+A-N+2}[\Gamma(N / 2)]^{2} \Gamma\left(\frac{2+m+A-N}{2}\right) \Gamma(2+r) \Gamma(2-N+r)}{\Gamma(4-N+2 r)} .(9)
\end{aligned}
$$

We invert the Mellin transform with a contour integral:

$$
F(q)=\frac{1}{2 \pi i} \int_{-i \infty}^{i \infty} d N F(N) Q^{-N}
$$

The contour in the complex $N$ plane runs in the strip of real- $N$ where $F(N)$ converges. Deforming the contour about singularities one-by-one generates an asymptotic series in $Q$. We use notation $F(Q) \leq Q^{-P}$ to indicate dependence falling at least as fast as $Q^{-P}$.

\subsection{HHC as Inequality}

Dependence at large $Q$ now comes from singularities to the right of the convergence strip. There are two distinct types.

The singularities of $\Gamma\left(\frac{2+m+A-N}{2}\right)$ are simple poles at

$$
N=2+m+A+2 K, \quad K=0,1,2 \ldots
$$

These are exactly the singularities creating the naive power counting of $H H C$. The existence of these singularities implies

$$
F(Q) \leq Q^{-m-A-2} \quad \text { (hhcregion only) }
$$

from these singularities alone, and barring a zero factor canceling the poles. As expected $b^{m} \rightarrow Q^{-m}$ : here the $H H C$ results are reproduced.

The dominant power $r$ locates the other singularities, seen in the pole from $\Gamma(2-N+r)$. These poles are independent of the $b^{m}$ dependence. This implies an additional contribution to power-behavior, not regulated by $b Q<1$. The poles and power behavior are

$$
\begin{array}{r}
N=2+r+K \quad K=0,1,2 \ldots \\
F(Q) \leq Q^{-2-r}, \quad(\text { dominant power region })
\end{array}
$$


regardless of the value of $m$. If such poles dominate, there is no power suppression of OAM $m \neq 0$.

As a result we have the following inequality for the large $Q$ dependence of the form factor,

$$
\begin{aligned}
& F(Q) \leq \frac{1}{1 / Q^{c+2}} \\
c= & M i n(r, m+A) .
\end{aligned}
$$

There is no power suppression of OAM $m \neq 0$ in general.

Rule of Thumb: We have extracted an improved $H H C$-rule of thumb: the asymptotic $Q^{2}$ dependence is determined by the minimum of powers $m, r$ in the integrands. The rule has been deliberately simplified to powers, suppressing logarithmic factors readily calculated, by further evaluating the Mellin inversion. These logarithms are obtained from the Mellin series and are a separate phenomenon from the asymptotic-freedom logarithms of pQCD. The origin of the inequality Eq. 13 is time-scale smearing: due to it, the suppression of OAM expected by naive counting is transferred to smearing inside the longitudinal wave functions, and helicity-flips can readily compete with helicity non-flip.

Effects of the Large $b$ Regions The singularities to the left of the convergence strip determine a series for small $Q \rightarrow 0$. These singularities are traced to the Gaussian model, Sudakov $b$-cutoff, or the large $b$ cutoff. We are not concerned with these singularities, showing that the large $b$ cutoff drops out of the power-laws stated above. However the numerical normalization of integrals depends on the cutoff method. It is a separate question needing separate analysis to find the numerical fit of models to data. Our numerical work is presented momentarily.

On this reasoning there is always a part of the calculation which is "strictly perturbative". We may calculate the large $b$ regions only with limited reliability: fine! The small $b$ regions known to be calculable behave as we claim, and are the only regions under conceptual dispute.

\subsection{Discussion}

As far as we know this is the first time that $H H C$ has been observed to fail for

large $Q^{2}$ form factors. Yet previous work has made related observations that 
the powers of $x$ can determine the $Q^{2}$ dependence. Feynman's mechanism 27. concentrated entirely on the region $x \rightarrow 1$ and ignored $b$. Feynman's work predated pQCD, and there are certainly regions in the $b$ integrals of that mechanism that would not be calculable, so the model is moot. Soper's 1977 paper [18] has a clear statement that the minimum power of $x$ or $k_{T}$ rules the results. The context there was the Drell-Yan-West [28] relation, which connects the $x \rightarrow 1$ behavior of inelastic structure functions with the $Q^{2}$ dependence of the form factor. Soper shows that one cannot prove the Drell-Yan-West relation deductively, but one can get an inequality and force the relation by choosing wave functions.

The literature is clouded here by treatments assuming that the two regions of $x \rightarrow 1$ and $b \rightarrow 0$ must give the same scaling, and forcing a result by circular logic. As we have just shown, the regions are different and no general rule can be made. Underlying this is the fact of perturbative calculability of short-transverse distance not being on the same footing as perturbative models of the $x \rightarrow 1$ dependence. For instance Brodsky and Lepage [11, 12] discuss the endpoint $x \rightarrow 1$ contribution of the proton form factor $F_{1}$. The authors argue that for the limit $x_{1} \rightarrow 1$, pQCD implies an $m=0$ wave function of order $\alpha_{s}^{2}$ perturbatively calculated to go like $\left(1-x_{1}\right)^{1}$. On this basis they find that a contribution to $F_{1}$ scaling like $1 / Q^{4}$ independent of the powers of $b$ inside the integrals. The $Q$ dependence of this contribution is same as that obtained from the $b \rightarrow 1 / Q$ region. Although perturbative analysis is not a valid approach to calculating wave functions, the result is consistent with ours.

Our results are due to time-scale smearing and should not be attributed to "end-point singularities" as the term is commonly used. Inside the hard scattering kernels in pQCD are combinations of inverse powers of $x$ or $1-x$. We are not exploiting these inverse powers in the evaluation of the Mellin moments. Our basic point is that the $x \rightarrow 1$ limit of the $m \neq 0$ wave function is not known. This uncertainty leads to the inequality given in Eq. 13. The end point singularities, when present, further enhance the end point contributions and expand the range of allowed values of $r$, the dominant power of $x$, for which there is no power suppression of OAM. We are also not concerned with endpoint wave functions (a la CZ) for the same reason: their relevance or lack of it is another subject.

To summarize the logic so far, for broad classes of $x$ dependence of the wave functions, which is unknown, the non-rule of HHC is transformed to a rule of HHNC, hadron helicity non-conservation. This is a new asymptotic 
prediction, and proves that $Q F_{2}\left(Q^{2}\right) / F_{1}\left(Q^{2}\right) \sim$ const can be an outcome of the theory up to the highest $Q$. To be equally fair, different broad classes of wave functions give $Q^{2} F_{2}\left(Q^{2}\right) / F_{1}\left(Q^{2}\right) \sim$ const. Our primary accomplishment enlarges the sphere of allowed possibilities. Measurements are still needed to determine what protons are.

\section{Numerical Studies, Sub-Asymptotic}

The scaling rule $Q F_{2}\left(Q^{2}\right) / F_{1}\left(Q^{2}\right) \sim$ const describes experiments at laboratory $Q$-values very far from asymptotic. We explored this region numerically.

\subsection{Studies with the Pion}

The pion provides a simple test system. We test the dominant integration regions by inserting factors of $b^{m}$ in the integrands, corresponding to $m$-units of OAM. The moment $\langle b(Q)\rangle_{\pi}$ is defined by

$$
<b(Q)>_{\pi}=\frac{\int d x d x^{\prime} d^{2} b b \mathcal{F}_{\pi}\left(Q, x, x^{\prime}, b\right)}{F_{\pi}\left(Q^{2}\right)} .
$$

The pion form factor $F_{\pi}\left(Q^{2}\right)$ is given by

$$
F_{\pi}\left(Q^{2}\right)=\int d x d x^{\prime} d^{2} b \mathcal{F}_{\pi}\left(Q, x, x^{\prime}, b\right)
$$

with the kernel $\mathcal{F}_{\pi}\left(Q, x, x^{\prime}, b\right)$ defined by

$$
\mathcal{F}_{\pi}\left(Q, x, x^{\prime}, b\right)=\int d x d x^{\prime} d b b \phi\left(x^{\prime}\right) \alpha_{s}(\mu) e^{-S\left(x, x^{\prime}, b, Q\right)} K_{0}\left(\sqrt{x x^{\prime}} b Q\right) \phi(x) \Phi(b) .
$$

Here $S\left(x, x^{\prime}, b, Q\right)$ is the Sudakov form factor. A model soft wavefunction $\Phi(b)=\exp \left(-b^{2} / 2 a^{2}\right)$ is also included, where the parameter $a=1 / \Lambda_{Q C D}$.

In Fig. 1 we plot the moment $\langle b(Q)\rangle_{\pi}$ using a wave function of the form

$$
\phi(x) \sim[x(1-x)]^{\delta},
$$

We study values of $\delta=0.2,1$, with and without the Sudakov form factor. In the latter case the momentum scale $\mu=Q / 4$ of the strong coupling is imposed, and the $b$ integrals are cut off at $b=1 / \Lambda_{Q C D}$. From the Figure it is clear that for $Q^{2}<100 \mathrm{GeV}^{2}$ the moment falls much slower than the asd prediction of $1 / Q$, regardless of the $x$-dependence of the wave function used. 


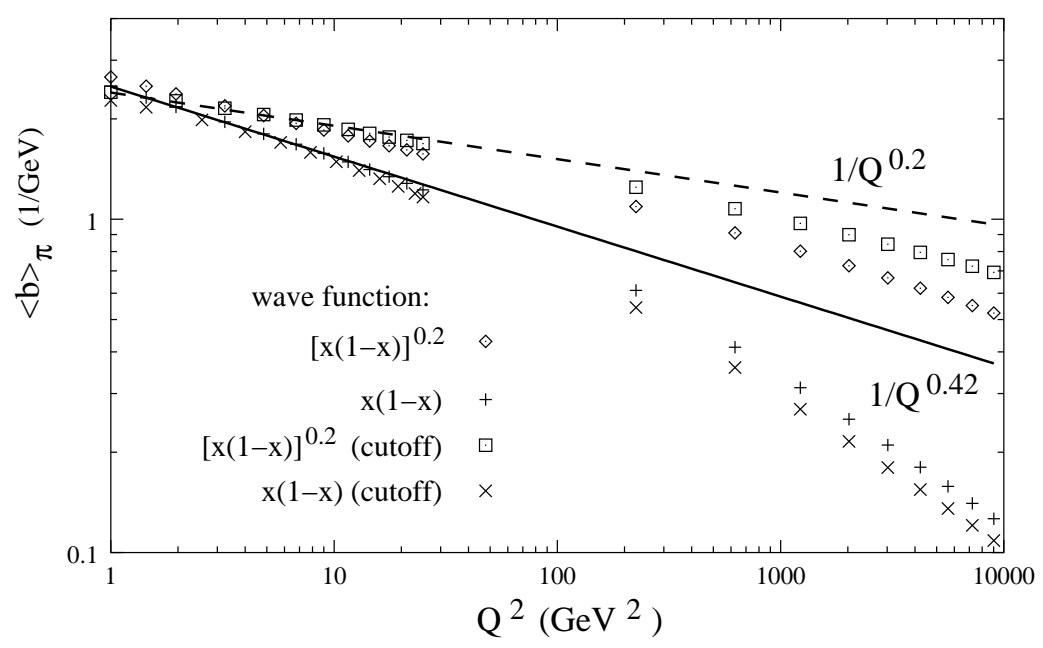

Figure 1: The moment $\left\langle b(q)>_{\pi}\right.$ of the pion form factor kernel, as defined in the text, using the $[x(1-x)]^{\delta}$ form of wave function for $\delta=0.2,1$. The moment decreases with $Q$ much slower than the $1 / Q$ behaviour expected in the asd HHC model. Results are shown with and without including the Sudakov effects. The solid $\left(1 / Q^{0.42}\right)$ and the dashed $\left(1 / Q^{0.2}\right)$ line represent a simple power law fit at small $Q^{2}$ for $\delta=1$ and $\delta=0.2$ respectively. 


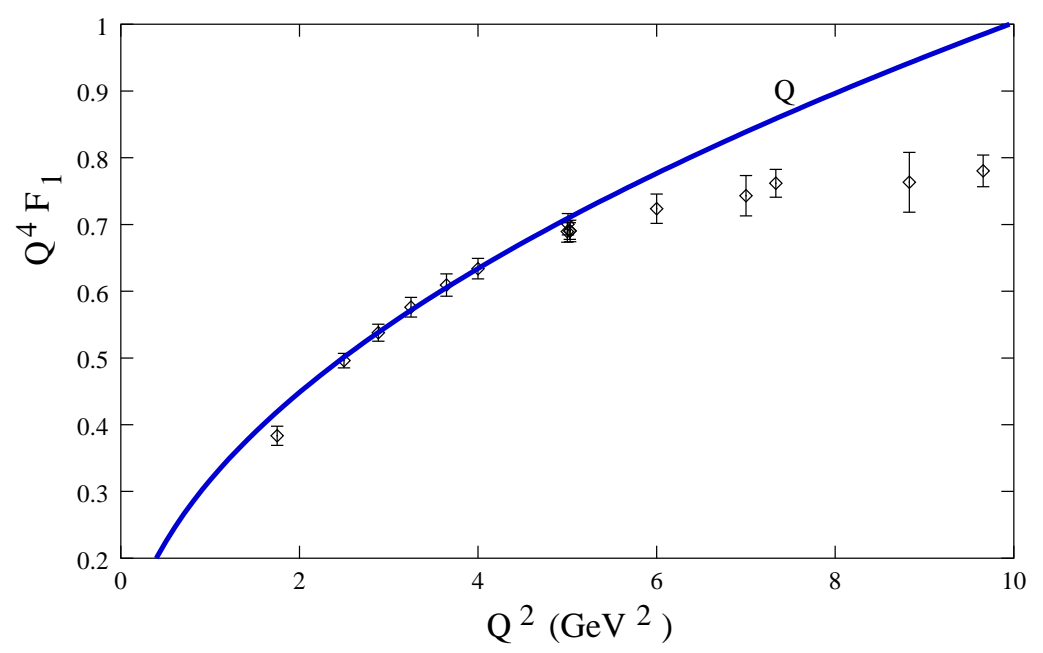

Figure 2: The proton form factor $F_{1}$ for moderate $Q^{2}$, extracted using the Jlab data for $G_{E} / G_{M}$ and the SLAC data for $G_{M}$. The ratio $G_{E} / G_{M}$ is obtained from the parameterization [2] $\mu_{p} \frac{G_{E}}{G_{M}}=1-0.13\left(Q^{2}-0.04\right)$. The solid line represents $F_{1} \sim 1 / Q^{3}$.

\subsection{Studies with the Proton}

We turn to the proton form factors. We note that the JLAB data for $Q F_{2} / F_{1}$ is flat even below the $Q$-range where $F_{1} \sim 1 / Q^{4}$ begins to fit. In Fig. 2 we show a plot of $Q^{4} F_{1}$. The solid line in the plot corresponds to the behaviour $F_{1} \sim 1 / Q^{3}$. It is clear from the plot that the scaling $F_{1} \sim 1 / Q^{4}$ is seen only for $Q^{2} \geq 6 \mathrm{GeV}^{2}$, which is larger than the momentum regime explored at JLAB so far. This is cause for concern. It is nevertheless entirely possible that the scaling observed in the ratio is not overly sensitive to $Q^{2}$, and will continue to larger $Q^{2}$. We investigate this in greater detail numerically.

To probe the dominant integration regions, we turn to calculating $b$ - moments of the proton form factor kernel. These are multidimensional integrals of which our analysis in the previous section determines but a lowdimensional strip. We spare the reader a listing of dozens of Feynman diagrams and substantially complicated kernels listed in the literature [23]. The form factor $F_{1}$ in impact parameter coordinates can be written symbolically 


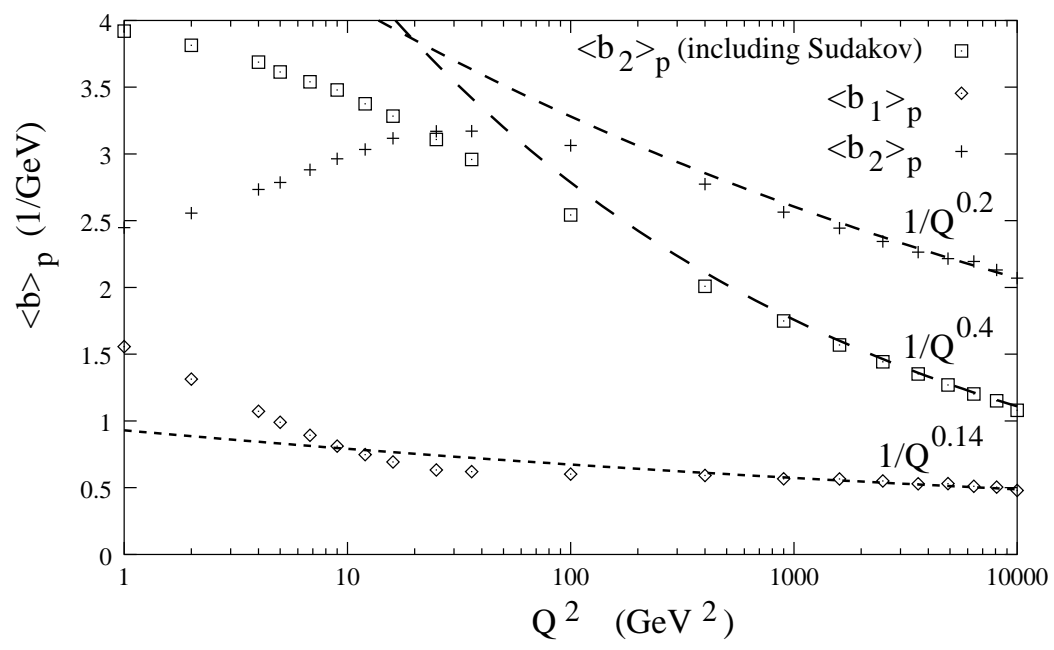

Figure 3: The moment of the transverse separation $b_{1}$ and $b_{2}$ for proton using the wave function of the form $\left(x_{1} x_{2} x_{3}\right)^{0.5}$. The $<b_{2}>_{p}$ moment is shown both with and without including the sudakov form factor. The small dashed $\left(1 / Q^{0.14}\right)$, medium dashed $\left(1 / Q^{0.2}\right)$ and the large dashed $\left(1 / Q^{0.4}\right)$ lines represent simple power law fits at large $Q^{2}$. 
as 23

$$
\begin{aligned}
F_{1}\left(Q^{2}\right)= & \sum_{j=1}^{2} \frac{4 \pi}{27} \int_{0}^{1}(d x)\left(d x^{\prime}\right) \int_{0}^{\infty} b_{1} d b_{1} b_{2} d b_{2} \int_{0}^{2 \pi} d \theta\left[f_{N}(w)\right]^{2} \\
& \times \tilde{H}_{j}\left(x_{i}, x_{i}^{\prime}, b_{i}, Q, t_{j 1}, t_{j 2}\right) \Psi_{j}\left(x_{i}, x_{i}^{\prime}, w\right) \\
& \times \exp \left[-S\left(x_{i}, x_{i}^{\prime}, w, Q, t_{j 1}, t_{j 2}\right)\right]
\end{aligned}
$$

with $(d x)=d x_{1} d x_{2} d x_{3} \delta\left(1-x_{1}-x_{2}-x_{3}\right)$. The variable $\theta$ is the angle between $\mathbf{b}_{1}$ and $\mathbf{b}_{2}$ and $x_{i}$ and $x_{i}^{\prime}$ refer to the initial and final $x$ variables. The expressions for the hard scattering $\tilde{H}_{j}$, the Sudakov form factor $S$, and function $\Psi_{i}$ are given in Ref. [23]. Now defining

$$
F_{1}(Q)=\int b_{1} d b_{1} b_{2} d b_{2} \mathcal{F}_{P}
$$

from Eq. [17, we define moments $<b_{j}(Q)>_{p}$ as follows:

$$
<b_{j}(Q)>_{p}=\frac{\int b_{1} d b_{1} b_{2} d b_{2} \mathcal{F}_{P} b_{j}}{F_{1}\left(Q^{2}\right)}
$$

The function $\Psi_{i}$ is where the linear combinations of the products of initial and final $x$ wave functions are found. The most singular part of the kernel in the limit $x_{1} \rightarrow 1$ and $x_{1}^{\prime} \rightarrow 1$ is obtained from the $\tilde{H}_{1} \Psi_{1}$ term, which is of the form,

$$
\tilde{H}_{1} \Psi_{1} \sim \frac{K_{0}\left(\sqrt{\left(1-x_{1}\right)\left(1-x_{1}^{\prime}\right)} Q b_{1}\right) K_{0}\left(\sqrt{x_{2} x_{2}^{\prime}} Q b_{2}\right) \phi\left(x_{i}\right) \phi\left(x_{i}^{\prime}\right)}{\left(1-x_{1}\right)\left(1-x_{1}^{\prime}\right)}
$$

Here $K_{0}$ is the modified Bessel function of order zero.

For the test we explore a wave function $\phi\left(x_{i}\right)$ given by

$$
\phi\left(x_{i}\right) \sim\left(x_{1} x_{2} x_{3}\right)^{\delta} .
$$

The numerator in $\Psi_{1}$ is then proportional to $\left(x_{1} x_{2} x_{3} x_{1}^{\prime} x_{2}^{\prime} x_{3}^{\prime}\right)^{\delta}$. The Bessel functions imply that in the limit of large $Q,\left(1-x_{1}\right)\left(1-x_{1}^{\prime}\right) b_{1}^{2} \rightarrow 1 / Q^{2}$ and $x_{2} x_{2}^{\prime} b_{2}^{2} \rightarrow 1 / Q^{2}$. As long as $\delta \leq 0.5$ the time-scale smearing will dominate. For $\delta=0.5$ we get $F_{1} \rightarrow 1 / Q^{4}$ even though a dominant region in $b_{1}$ and $b_{2}$ is independent of $Q$ ! For $\delta \leq 0.5$ the moments of $b_{1}$ and $b_{2}$ should also have a region independent of $Q$. 
We check these predictions by performing the calculation using $\delta=0.5$. We ignore the Sudakov form factor for this test, because it is a side issue. We evaluate the strong coupling at $Q / 4$. In figure 3 we plot the moment of the transverse separations $b_{1}(Q)$ and $b_{2}(Q)$ as a function of $Q$. We find that for $Q^{2}>100$, the moments $<b_{2}>_{p}$ and $<b_{1}>_{p}$ fall asymptotically as $1 / Q^{0.2}$ and $1 / Q^{0.14}$ respectively, in agreement with our analytic expectations. If we include the Sudakov form factor the moment $<b_{2}>_{p}$ falls as $1 / Q^{0.4}$, asymptotically. This is only a slightly stronger decay compared to the earlier case. In contrast to earlier expectations, the Sudakov form factor does not much suppress the importance of the end-point region.

The results with end point dominated COZ [29, 30] $x$-dependence are similar. In this case, as shown in Fig. [4 we find that the $\left\langle b_{2}\right\rangle_{p}$ decays very slowly with $Q$ for a wide range of $Q$. This is quite interesting, because, as shown in Fig. 5 , the form factor $F_{1}$ itself does not show good $1 / Q^{4}$ scaling in this momentum regime, if the sudakov form factor is not included. The calculation indicates that the scaling seen in the moment, and hence the ratio $Q F_{2} / F_{1}$, is more general than that seen in $F_{1}$. This is not totally unexpected in a ratio. At very large $Q$ it starts to fall faster, but only as $1 / Q^{0.6}$ : well below the supposed $1 / Q$ rule of asd assumptions.

\subsection{The Ratio $Q F_{2}\left(Q^{2}\right) / F_{1}\left(Q^{2}\right)$}

Based on these studies we come to a prediction for the ratio $Q F_{2}\left(Q^{2}\right) / F_{1}\left(Q^{2}\right)$. This prediction is based on power-counting, and the dominant integration regions probed by the moments. It is as complete a prediction as now possible, taking into account that one unit $\Delta m=1$ of orbital angular momentum is needed for $F_{2}$ to proceed for $Q^{2}>>\mathrm{GeV}^{2}$. Up to small and model dependent corrections, of order $Q^{0.05}$, the power counting gives the scaling behavior but not the normalization of the data, via the relation

$$
<b_{2}(Q)>_{p} \sim \frac{Q F_{2}\left(Q^{2}\right)}{F_{1}\left(Q^{2}\right)} \sim \text { constant. }
$$

What if this prediction fails? It can fail, according to our asymptotic studies, if the dominant power $r$ is "large". Large $Q^{2}$ studies of $F_{2}$ not only probe quark OAM, but also they can tell us details of the dominat $x$ power associated with $m \neq 0$. 


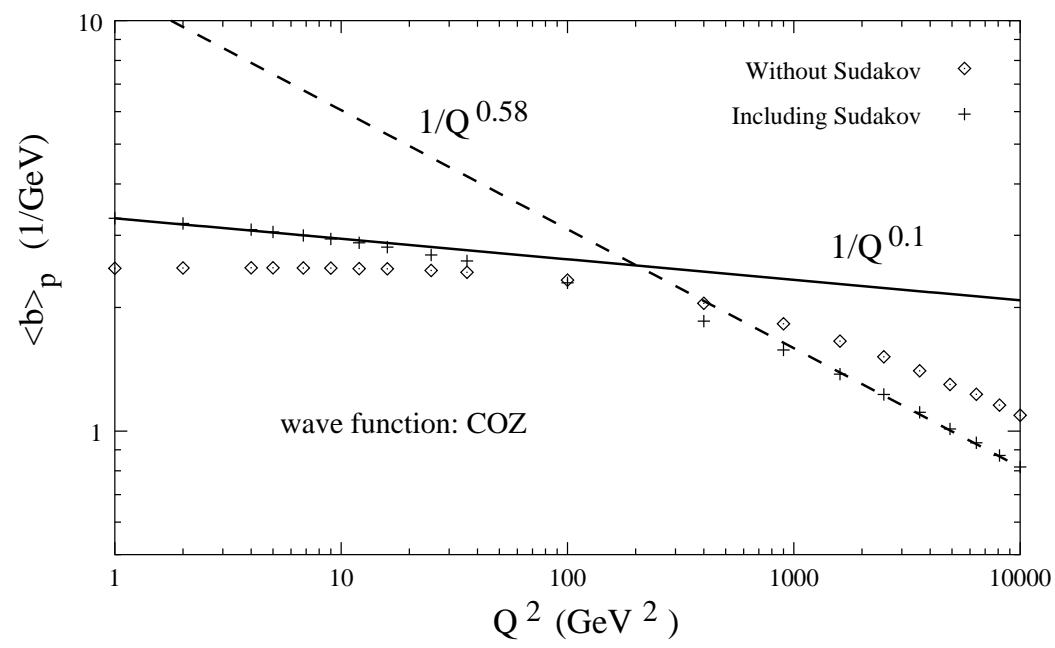

Figure 4: The moment of the transverse separation $<b_{2}(Q)>_{p}$ for the proton form factor kernel using the COZ wave function [29]. The solid $\left(1 / Q^{0.1}\right)$ and dashed $\left(1 / Q^{0.58}\right)$ lines represent simple power law fits at small and large $Q^{2}$ respectively. The dependence on $Q$ is much weaker than predicted by asd relations, and supports a flat prediction for $Q F_{2} / F_{1}$ at JLAB momentum transfers 


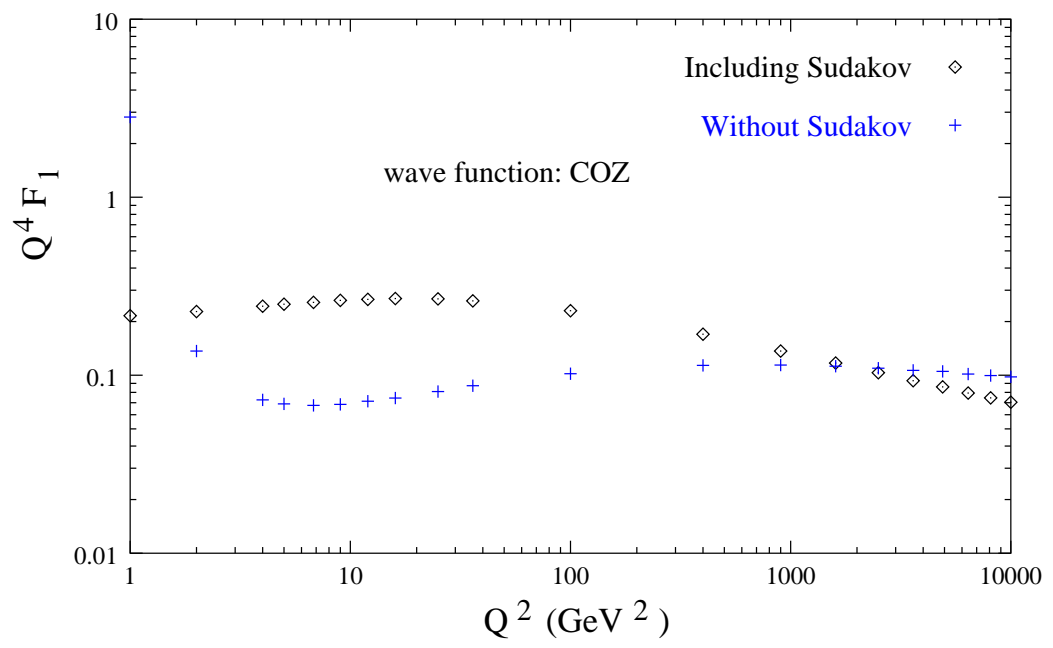

Figure 5: The $Q^{2}$ dependence of the calculated proton form factor $F_{1}$ using the COZ wave function [29. Results are shown both with and without including the Sudakov form factor. Details are in the text.

\subsection{Positivity of $G_{E}$}

In the Appendix of a 1959 Reviews of Modern Physics article 31] there are listed two form factors denoted $A$ and $B$, now called $G_{E}$ and $G_{M}$, which are linear combinations of Rosenbluth's:

$$
\begin{array}{r}
G_{M}=F_{1}+\kappa F_{2} ; \\
G_{E}=F_{1}-\kappa \tau F_{2} ; \\
\tau=\frac{Q^{2}}{4 m_{p}^{2}}
\end{array}
$$

and $\kappa$ is the anomalous magnetic moment. For some reason these are called the Sachs form factors 32. Yennie et al gave 31] the alternative linear combinations simply to emphasize that the definitions of form factors was arbitrary.

It is interesting to observe that if the trend of $Q F_{2}\left(Q^{2}\right) / F_{1}\left(Q^{2}\right) \sim$ const continues, the value of $G_{E}$ will reach zero, and cross to a negative value. One wonders 33] whether there is a physical significance and a barrier to this unusual occurence. The meaning of $G_{E}$ is angular momentum $\Delta J_{z}=0$ 
spin-preservation in the Briet frame. If $G_{E}=0$ the proton spin must flip in the scattering, which seems industrially useful. ${ }^{4}$ We find nothing special about this. The amplitudes in the spacelike region cannot be limited further than general principles of analyticity, Lorentz and gauge invariance, and so on. It is perfectly consistent to arrange timelike discontinuities so that the sign change of $G_{E}$ occurs without violating anything holy.

\section{Concluding Remarks}

Our calculations show that the (newly revised) asymptotic behaviour of powers of $b$ is achieved only at very large $Q^{2}$. Physics has many asymptotic predictions, which by construction have suppressed information needed to know when they will apply. To repeat: methods inherent in asymptotic prediction strongly tend not to tell you where the prediction will be valid. In QCD it was early thought the "asymptotic" regime had to occur at energies very large compared to $\Lambda_{Q C D}$, the strong scale. This was built wrongly into dogma. Very often asymptotically large logarithms are needed to justify some approximation. We have shown here that the scales of asymptopia for form factors are vastly beyond experimental comparisons. Asymptopia is effectively meaningless, because all the new physics of very high energies has been left out.

Meanwhile, the finite $Q^{2}$ effects of OAM are hardly suppressed at all. They are suppressed in explicit calculations by even less than the revised asymptotic behavior. The pyramid of assumptions that the $s$-wave distribution amplitudes are meaningful falls into very grave doubt. To put this more directly, we don't have a reason to use a distribution amplitude any more.

The usual approach to pQCD, in which wave functions are assumed to be unknown, predicts HHNC: hadron-helicity non-conservation. There is an inequality that helicity-flip processes do not lead but can have equal power with helicity non-flip processes.

The experimental observation of $R(Q)=Q F_{2} / F_{1}$ appears to have a dual meaning. The ratio is a very robust quantity, which remains flat even in the regime where $F_{1}$ is not clearly dominated by quark-counting. The same ratio is an important asymptotic quantity, which says things about the wave functions. The common notion that proton wave functions are "cubic",

\footnotetext{
${ }^{4}$ Quantum computing, which often invokes high-speed, high-precision spin-flips, may or may not ever need the physics of multi-GeV spin flips, but it stands ready.
} 
namely going like $x_{1} x_{2} x_{3}$, is ruled out for wave functions calculating $F_{2}$. Applications of the $\mathrm{CZ}$ wave functions are ruled out for $F_{2}$, if the ratio $R$ continues to be flat at large $Q^{2}$.

Predictions for protons are mirrored in predictions of the same type for neutrons. If the flat $R(Q)$ ratio is followed for neutrons, then the hallowed Galster fits 34 to neutron data will eventually fail 33. We predict a flat $R$ ratio for neutrons on the basis of isospin independence of QCD.

The study of polarization transfer in large nuclei, $\vec{e}\left(A, e^{\prime}\right) \vec{p}^{\prime}$ should yield further information. If the existing form factors are dominated by asd, which we do not believe but is still worth testing, then nuclear filtering will not make the distances shorter, and the ratio $R$ should be flat with $A>>1$. The regime of small $A \sim 10$ has no advantages and many complications due to few-body effects, and predictions are more difficult. If the existing form factors are dominated by quark mass effects, then nuclear filtering will not make any difference and the ratio $R$ should again be flat. If quark OAM is responsible for the flatness of $R$ as we believe, then filtering will kill the large transverse extent of large OAM [35, 36, $F_{2}$ should be depleted relative to $F_{1}$, and $R$ will decrease with $A>>1$. We intend to dedicate a study to quantifying these predictions.

JLAB has made a pivotal experimental discovery which will be a permanent subject of discussion. The experimentally observed flatness of $Q F_{2} / F_{1}$ is a signal of substantial quark orbital angular momentum in the proton. Higher momentum transfer measurements would be helpful in confirming this interpretation. The numerical size of $R$ cannot be converted directly to a wave function, because it is only a single number. But the value of $R$ can rule out models which omit quark OAM. Further studies at higher $Q^{2}$ may separate constituent quark models with OAM [6], which tend to have a scale (the quark mass) forcing turn-over of $R$ with increasing $Q^{2}$.

Acknowledgements: Work supported by Department of Energy Grant Number DE-FG03-98ER41079.

\section{References}

[1] M.K. Jones et al, Phys. Rev. Lett. 84, 1398 (2000).

[2] O. Gayou et al, Phys. Rev. Lett. 88, 092301, (2002). 
[3] For example, A. Salam endorsed $Q^{2} F_{2} / F_{1} \sim$ const in 1963, when Hofstadter's data was pre-emininent, without quite explaining why. See International Conference on Nuclear Structure, Eds. R. Hofstadter and L. I. Schiff, Stanford (1963).

[4] R. Buniy, J. P. Ralston, and P. Jain, in VII International Conference on the Intersections of Particle and Nuclear Physics (Quebec City, 2000) edited by Z. Parseh and W. Marciano (AIP, NY 2000), hep-ph/0206074.

[5] J. P. Ralston, R. V. Buniy and P. Jain Proceedings of DIS 2001, 9th International Workshop on Deep Inelastic Scattering, Bologna, 27 April - 1 May, 2001, hep-ph/0206063.

[6] M. R. Frank, B. K. Jennings and G.A. Miller, Phys. Rev. C54, 920 (1996); G.A. Miller and M. R. Frank, nucl-th/0201021.

[7] S. J. Brodsky and G. P. Lepage, Phys. Rev. D 24, 2848 (1981).

[8] P. Jain and J. P. Ralston, in Future Directions in Particle and Nuclear Physics at Multi-GeV Hadron Beam Facilities (Proceedings of the Workshop held at BNL, 4-6 March, 1993), hep-ph/9305250.

[9] X. D. Ji, J. Tang and P. Hoodbhoy, Phys. Rev. Lett. 76, 740 (1996); hep-ph/9510304.

[10] S. J. Brodsky and G. R. Farrar, Phys. Rev. D 11, 1309 (1975); V. A. Matveev, R. M. Muradian and A. N. Tavkhelidze, Lett. Nuovo Cim. 7, 719 (1973).

[11] G. P. Lepage and S. J. Brodsky, Phys. Rev. D 22, 2157 (1980).

[12] S. J. Brodsky and G. P. Lepage, Perturbative Quantum Chromodynamics, Ed. by A.H. Mueller, World Scientific, 1989.

[13] A. V. Belitsky, X. Ji and F. Yuan, hep-ph/0212351.

[14] C. R. Ji and A. F. Sill, Phys. Rev. D 34, 3350 (1986).

[15] N. Isgur and C. Llewelyn-Smith, Phys. Rev. Lett. 52, 1080 (1984).

[16] A.V. Radyushkin, Phys. Rev. D58, 114008 (1998). 
[17] A. Wijesoriya, et al Phys. Rev. Lett. 86, 2975 (2001); R. Gilman, J. Phys. G 28, R37 (2002).

[18] D. E. Soper, Phys. Rev. D 15, 1141 (1977)

[19] M. Diehl, Th. Feldmann, R. Jacob and P. Kroll, Nucl. Phys. B 596, 33 (2001).

[20] J. Botts and G. Sterman, Nucl. Phys. B325, 62 (1989).

[21] J. P. Ralston and B. Pire, Phys. Rev. Lett. 65, 2343 (1990).

[22] H.-N. Li and G. Sterman, Nucl. Phys. B 381, 129 (1992).

[23] H.-N. Li Phys. Rev. D 48, 4243 (1993).

[24] J. Bolz, R. Jakob, P. Kroll, M. Bergmann, and N. G. Stefanis, Z. Phys. C 66, 267 (1995).

[25] T. Gousset, B. Pire and J. P. Ralston, Phys. Rev. D53, 1202 (1996).

[26] B. Kundu, H.-N. Li, J. Samuelsson and P. Jain, hep-ph/9806419, Euro. Phys. Journal C 8, 637 (1999).

[27] R. P. Feynman, Photon-Hadron Interactions, Addison Wesley, 1970.

[28] S. D. Drell and T. M. Yan, Phys. Rev. Lett. 24, 181 (1970); G. W. West, Phys. Rev. Lett. 24, 1206 (1970).

[29] V. L. Chernyak, A. A. Ogloblin, I. R. Zhitnitsky Sov. J. Nucl. Phys. 48, 536 (1988); Yad. Fiz. 48, 841 (1988).

[30] V. L. Chernyak and A. R. Zitnitsky, Phys. Rep. 112, 173 (1984); Nucl. Phys. B 246, 52 (1984).

[31] D. Yennie, M. Ravenhall, and M. Levy, Rev. Mod. Phys 29, 144 (1957).

[32] W. Ernst, K. Wali, and M. Sachs, Phys. Rev. 119, 1105 (1960); ibid 126, $2256(1962)$

[33] We thank Charles Perdrisat for discussions on this point.

[34] S. Galster, H. Klein, J. Moritz, K.H. Schmidt, D. Wegener, and J. Bleckwenn, Nucl. Phys. B 32, 221 (1971). 
[35] P. Jain, B. Pire and J. P. Ralston, Phys. Rep. 271, 67 (1996).

[36] J. P. Ralston and B. Pire, "Color Transparency in Electronuclear Physics" in Proceedings of Second Workshop on Hadronic Physics with Electrons Beyond $10 \mathrm{GeV}$ (Dourdan, France 1990) Nuc. Phys. A 532, 155c (1991). 